\title{
Simulated tomographic reconstruction of ocean features using drifting acoustic receivers and a navigated source
}

\author{
Timothy F. Duda, Richard A. Pawlowicz, a) and James F. Lynch \\ Applied Ocean Physics and Engineering Department, Woods Hole Oceanographic Institution, Woods Hole, \\ Massachusetts 02543
}

\author{
Bruce D. Cornuelle \\ Scripps Institution of Oceanography, University of California at San Diego, La Jolla, California 92093
}

(Received 13 January 1995; accepted for publication 12 May 1995)

\begin{abstract}
Numerically simulated acoustic transmission from a single source of known position (for example, suspended from a ship) to receivers of partially known position (for example, sonobuoys dropped from the air) are used for tomographic mapping of ocean sound speed. The maps are evaluated for accuracy and utility. Grids of 16 receivers are employed, with sizes of 150,300 , and $700 \mathrm{~km}$ square. Ordinary statistical measures are used to evaluate the pattern similarity and thus the mapping capability of the system. For an array of $300 \mathrm{~km}$ square, quantitative error in the maps grows with receiver position uncertainty. The large and small arrays show lesser mapping capability than the mid-size array. Mapping errors increase with receiver position uncertainty for uncertainties less than 1000-m rms, but uncertainties exceeding that have less systematic effect on the maps. Maps of rms error of the field do not provide a complete view of the utility of the acoustic network. Features of maps are surprisingly reproducible for different navigation error levels, and give comparable information about mesoscale structures despite great variations in those levels. (C) 1995 Acoustical Society of America.
\end{abstract}

PACS numbers: $43.30 . P c, 43.30 . Z k$

\section{INTRODUCTION}

Mapping heterogeneities of ocean temperature is one of the primary goals of ocean remote sensing using acoustic propagation, a procedure commonly known as acoustic tomography. One-way acoustic transmission from a few sources to many acoustic receivers is the simplest tomographic arrangement because of the technical simplicity and cost effectiveness of receivers relative to transmitters. Five "time-of-flight" tomography experiments have already produced three-dimensional maps of ocean temperature structure: a 1981 pilot experiment with four sources and five receivers in the North Atlantic (Chiu and Desaubies, 1987; Cornuelle et al., 1985), the tomography component of the Greenland Sea Project (Worcester et al., 1993), a Gulf Stream synoptic study (Chester et al., 1994) a Mediterranean deep convection study (Send et al., 1994), and the Acoustical Mid-Ocean Dynamics Experiment (Dushaw et al., 1994).

Acoustic propagation in these experiments, at carrier frequencies between 250 and $500 \mathrm{~Hz}$, was shown to be consistent with geometrical optics (the ray approximation), which is strictly valid only for infinitesimal wavelength but otherwise independent of frequency. Time-of-flight tomography deduces the sound-speed field from measured acoustic travel times over known ray paths. Tomography equipment is most often tethered to a mooring or a ship, rather than affixed to the seafloor, to reduce reflections from poorly known bathymetry and to provide the widest distribution of multipath arrivals, both strongly and weakly confined to the sound-channel minimum. Localization or "navigation" of

a) Now at Institute of Ocean Sciences, Sidney, BC, Canada. V8L 4B2. this slightly mobile equipment better constrains the paths and reduces errors. This navigation usually requires emplacement of short-range acoustic devices to track the instruments. In practice, bottom reflected receptions are excluded from consideration.

There is a simpler alternative to this fully constrained time-of-flight tomographic arrangement. Ocean sound-speed structure can be estimated using acoustic arrival structures, without attempting to determine the mean sound speed. Information about the ocean structure is contained in the pattern of arrivals, relative to an arbitrary reference time, and the pattern is easily recorded without measuring absolute distance or travel time. The resulting measurement of structure can be referred to as "relative arrival tomography." Navigational constraints are eased; but not eliminated. Raygeometry fundamentals (i.e., number of turning points of rays, initial angle, etc.) must be properly identified, however, so position knowledge to a few kilometers must still be known, requiring minimal navigation by modern standards.

The availability of inexpensive satellite navigation receivers (GPS, Global Positioning System) allows navigation of buoys to within hundreds of meters at a minimum, and to better precision if differential GPS or other GPS enhancements are utilized. This means that drifting receivers can monitor and transmit their own positions, along with acoustic tomography signals. However, the positions of suspended hydrophones cannot generally be known to the precision required for absolute time-of-flight tomography (tens of meters) without using a more expensive and elaborate system. We are investigating the utility of receivers costing less than $\$ 5000$, modified sonobuoys, or other simple drifters. 
The effectiveness of tomography with untracked or moving instruments has already been analyzed in two studies (Cornuelle, 1985; Gaillard, 1985). Those studies have shown that the arrival patterns contain useful information about the ocean structure. The ocean reconstruction simulations shown here are similar to those of Comuelle (1985), who considered a single configuration of sources and receivers. This work extends Cornuelle's analysis by comparing reconstructions from simulated arrays of many s.zes, and evaluates the results in terms of propagation geometry and ocean scale lengths. Each array to be considered has a single source and many receivers. The source positions are assumed to be knowable in the field, thus their positions are known in the simulations. Receivers have position uncertainties of up to $4.0 \mathrm{~km}$. Acoustic receivers of this type may include rapidly deployable expendable moorings (under development at WHOI) or short-lived expendable devices such as sonobuoys, surface drifting buoys with GPS receivers and freely dangling hydrophones, or specially equipped RAFOS floats (Rossby et al., 1986).

The simulations show that navigation errors of properly designed gridded arrays of receivers do not deteriorate tomographic estimates of sound-speed struclure unless they exceed $200 \mathrm{~m}$. Often, even larger errors of 1 or $2 \mathrm{~km}$ do not significantly affect the results. The hoizontal geometry and scale lengths of tomographic arrays, most effectively measured relative to the scale lengths of structures to be mapped, are qualitatively shown to more strongly effect the mapping ability than do $\mathrm{km}$-scale location uncertainties.

The simulation method is straightforward, using previously described techniques. A prescribed three-dimensional field of sound speeds, referred to as the simulated ocean, is used to generate simulated acoustic propagation data, which are then used to map the field. The similarity of the prescribed and mapped fields are evalualed with a number of statistical methods. The simulated ocean is described in Sec. I. The tomography array configurations are described in Sec. II. The tomographic mapping procedure is reviewed in Sec. III, which also describes specific details of the simulations. The maps are compared and statistically evaluated in Sec. IV. Section V is a summary.

\section{SIMULATED OCEAN}

The prescribed sound-speed field used here was distributed to various researchers by C.-S. Chiu to allow simulated benchmark testing of ocean tomography techniques at the 124th Meeting of the Acoustical Sociery of America (Lynch et al., 1992). This three-dimensional scalar field is intended to represent midlatitude, mid-gyre ocean sound-speed structure, with horizontally homogeneous and isotropic soundspeed perturbations. The field is the sum of a layered mean sound-speed structure which we call the reference ocean (Fig. 1), and a three-dimensional field of mesoscale baroclinic structures. The mesoscale perturbation field is derived from four baroclinic oscillation modes (Gill, 1982, Chap. 6). Sound-speed perturbation modes associated with those motions, each with maximum value unity, are shown in Fig. 2 . Each mode has horizontal position-dependent amplitudes which are consistent with a horizontally isotropic Gaussian

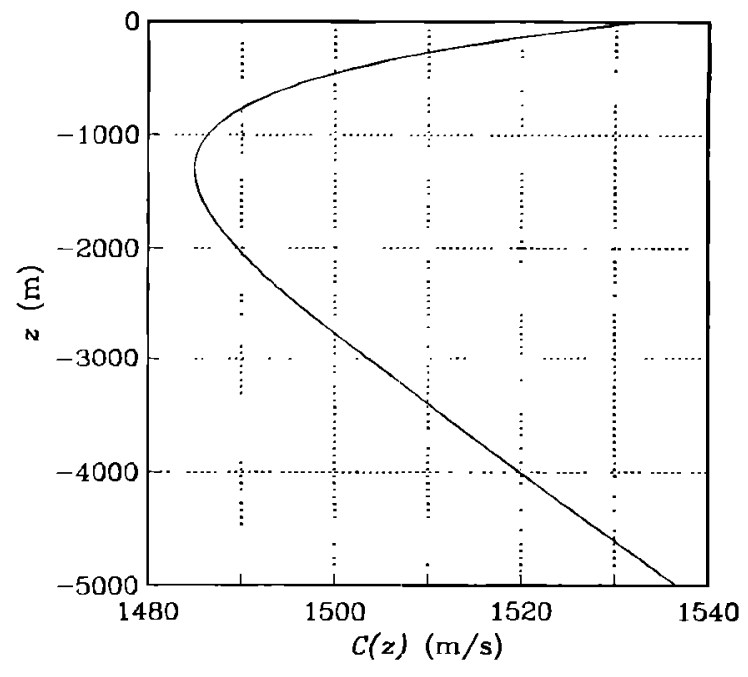

FIG. I. The mean sound-speed profile for the reference ocean, which is the layered component of the simulated ocean, is of the SOFAR channel midlatitude type.

covariance function of scale length $100 \mathrm{~km}$. Variances of the fluctuations of each of the modes (one through four), measured at the depths of maximum amplitude of each mode, are $7.3,11.5,9.0$, and $4.4 \mathrm{~m}^{2} \mathrm{~s}^{-2}$, respectively.

The sum of the squares of the modal shapes, weighted by the variances, gives the depth structure of sound-speed variance, shown in Fig. 3. The reduction of sound-speed variance to zero at the surface is an artifact of including only large-scale dynamical structures meeting rigid-lid boundary conditions, and would not occur if small-scale or thermohaline features were included. The maximum variance of about $26 \mathrm{~m}^{2} \mathrm{~s}^{-2}$ corresponds to an rms temperature fluctuation of about $1.7^{\circ} \mathrm{C}$. This is somewhat higher variance than often seen in the mid-Pacific, for example, also shown in Fig. 3. For this reason, simulations are also performed for three rescaled fluctuation fields whose variances are also shown in Fig. 3, obtained by multiplying sound-speed fluctuation amplitudes by $0.5,0.25$, and 0.125 .

The field domain is $1000 \mathrm{~km}$ square and $5 \mathrm{~km}$ deep. It is sampled at $25-\mathrm{km}$ horizontal and $50-\mathrm{m}$ vertical intervals.

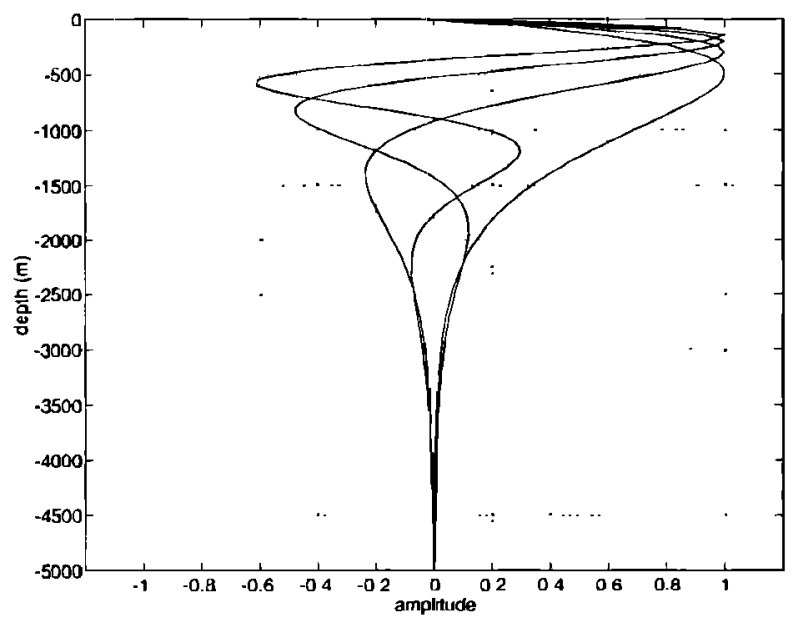

FIG. 2. The four sound-speed perturbation modes, associated with four baroclinic modes of vertical displacement, are mosl energetic in the upper 2 $\mathrm{km}$ of the water column. 


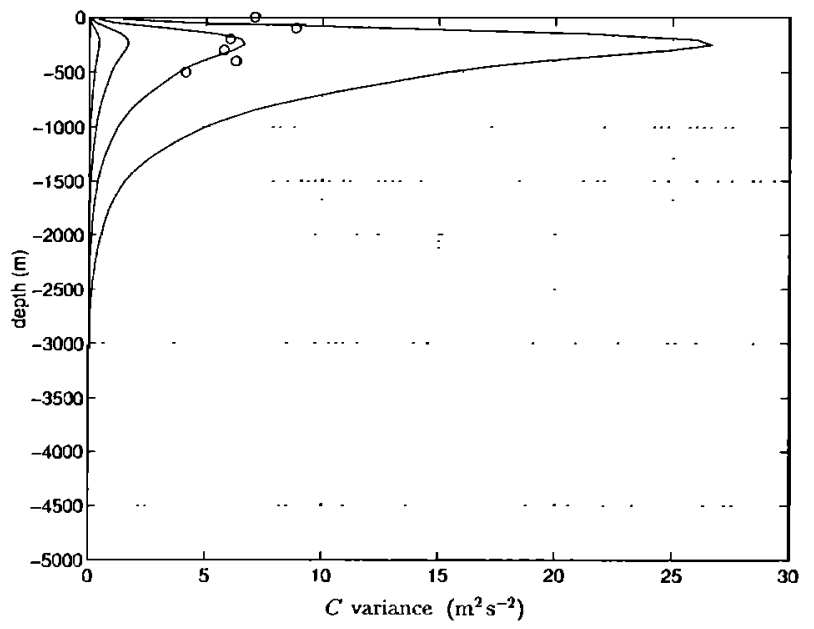

FIG. 3. The variance of sound speed in the simulated ocean is a strong function of depth. The right-most curve shows the variance of the originally provided field. The circles show variances from the upper mid-Pacific, collected during the Slice89 experiment (Duda et al., 1992). Since the simulated ocean had higher variance than these observations, the tomographic reconstructions were also tested for versions of the simulated ocean with perturbation amplitudes scaled down by one-half, one-quarter, and oneeighth. The associated variances of these versions are shown.

Figure 4 is a contour map of sound-speed perturbations at $700 \mathrm{~m}$ depth. The perturbations are less than $10 \mathrm{~m} / \mathrm{s}$. The perturbations have high vertical coherence, expected from the limited number of vertical modes. The Gaussiancorrelation nature of the perturbations gives a visual (synoptic) scale of 75 to $100 \mathrm{~km}$ to the patterns.

\section{ACOUSTIC ARRAY CONFIGURATIONS}

The mapping procedure is simulated for three array configurations and a variety of receiver location uncertainty variances. Receiver depths are assumed to be known. Statistics of map variability for each case are compiled by averaging a small number of realizations, typically four, each having independent, randomly generated receiver position errors.

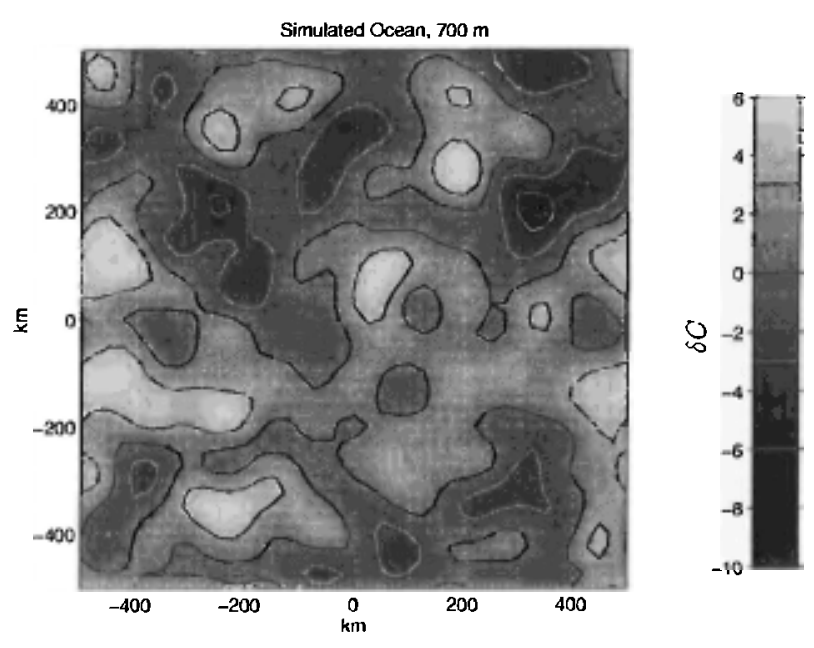

FIG. 4. The mesoscale perturbation sound-speed field is shown for the surface at $700 \mathrm{~m}$ depth. The perturbations range from about -7 to about $6 \mathrm{~m} / \mathrm{s}$. The perturbation correlation length scale of about $100-\mathrm{km}$ is apparent.

(a)

(b)

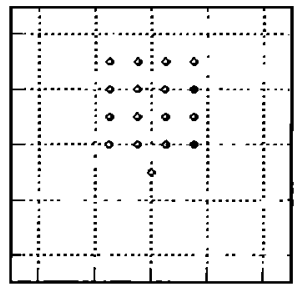

(c)

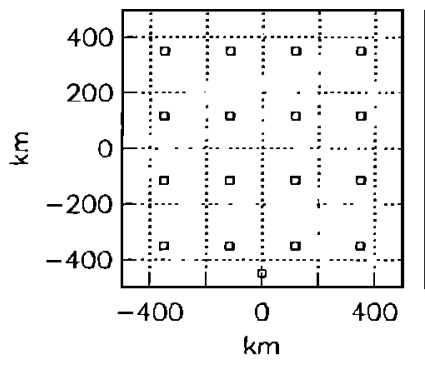

FIG. 5. (a) The 300-km array array baseline (default) position is shown. The square grid of 16 receivers is above the center of the $1000-\mathrm{km}$ square domain, and the single source is outside the grid. (b) Tomographic reconstructions were made with the array located at 12 additional positions, with receiver-grid centers indicated by the diamonds. The center for the baseline location (position 13) is also shown. Position 1 is at the upper left, 8 at the center right, 12 at the lower right, and so forth. (c) The $700-\mathrm{km}$ array positions are shown, with the source again located outside the receiver grid. (d) The 150-km array is shown, again with the source outside the grid.

The receiver arrays are square grids of 16 elements. The grid positions, $\mathbf{X}_{i}$, represent assumed receiver locations (the subscript indicates multiple position vectors in each array). The actual simulated receiver positions are nearby, at $\mathbf{X}_{i}+\mathbf{d}_{i}$, where $\mathbf{d}_{i}$ are position error vectors. The calculation method and properties of the $d_{i}$ are given in Sec. III. A single acoustic source is located outside of the array in each case, to eliminate short source/receiver transects to nearby grid elements having only a few (or zero) eigenrays.

Three array sizes are used: $150 \mathrm{~km}$ (50-km spacing), 300 $\mathrm{km}$ (100-km spacing), and $700 \mathrm{~km}$ (233-km spacing). The arrays and their positions are shown in Fig. 5. In each array, the acoustic source is positioned slightly outside of a square grid of receivers; it appears below the grid in the figures. Simulations using the $150-$ and $700-\mathrm{km}$ arrays were done with the array positions $\left(\mathbf{X}_{i}\right)$ fixed at single positions within the domain. The 300-km array is tested in a similar manner, and is additionally tested at 13 positions to investigate the mapping ability over a variety of of ocean features. The centers of the 13 position are shown in Fig. 5(b), with the square grid outlined for a few cases. The standard position of the $300-\mathrm{km}$ array is shown in Fig. 5, slightly above (north of) the center of the domain. Tested positions of the two other arrays are also shown. Table I lists all of the cases examined here.

In order to verify that suitable source and receiver depths were chosen, mapping sensitivity to instrument depth was tested for the standard-position $300-\mathrm{km}$ array prior to the main simulations. Three depth configurations were tested. The first had the source and all receivers at $1000-\mathrm{km}$ depth. The second had the source and receivers at $1300-\mathrm{m}$ depth, near the sound-speed minimum. The third configuration had 
TABLE I. The $x$ 's indicate which receiver displacement uncertainty values were used for various simulations. The array grid size and other indicators distinguish the runs. The column labeled $300 \mathrm{kn}$ (move) indicates testing of the $300 \mathrm{~km}$ array at various positions. The sprecial cases (ar) and (sr) are axial-depth $(1300 \mathrm{~m})$ instruments and shallow reveivers $(130 \mathrm{~m})$, respectively. The $300 \mathrm{~km}$ (redu) entries indicate those performed with attenuated versions of the ocean having $\frac{1}{4}, \frac{1}{16}$, and $\frac{1}{64}$ the baseline sound-speed variance.

\begin{tabular}{|c|c|c|c|c|c|c|}
\hline$\sigma_{d}(\mathrm{~m})$ & $\begin{array}{l}150 \\
\mathrm{~km}\end{array}$ & $\begin{array}{l}300 \\
\mathrm{~km}\end{array}$ & $\begin{array}{c}300 \\
\mathrm{~km} \text { (move) }\end{array}$ & $\begin{array}{c}300 \\
\mathrm{~km} \text { (rec'u) }\end{array}$ & $\begin{array}{c}300 \\
\mathrm{~km}(\mathrm{sr}, \mathrm{ar})\end{array}$ & $\begin{array}{l}700 \\
\mathrm{~km}\end{array}$ \\
\hline 0.01 & $x$ & $x$ & & & & $x$ \\
\hline 1 & $x$ & $x$ & & $x$ & & $x$ \\
\hline 10 & $x$ & $x$ & & & & $x$ \\
\hline 100 & $x$ & $x$ & & & & $x$ \\
\hline 200 & $x$ & $x$ & & & & $x$ \\
\hline 500 & $x$ & $x$ & & & $x$ & $x$ \\
\hline 1000 & $x$ & $x$ & $x$ & $x$ & & $x$ \\
\hline 1200 & $x$ & $\times$ & & & & $x$ \\
\hline 1500 & $x$ & $\times$ & & & & $x$ \\
\hline 2000 & & $x$ & & $x$ & & \\
\hline 2500 & & $x$ & & & & \\
\hline 3000 & & $x$ & & & & \\
\hline 3500 & & $x$ & & & & \\
\hline 4000 & & $x$ & & $x$ & & \\
\hline
\end{tabular}

the source at $1000 \mathrm{~m}$ and receivers at $130 \mathrm{~m}$, a typical hydrophone depth for expendable sonobuoy receivers. The three cases were compared, using techniques described in the next section, for the single case of $5100-\mathrm{m}$ rms receiver uncertainty. The second arrangement did not work quite as well as the first because some ray pairs that symmetrically cycled about the sound-channel minimum could not be reliably distinguished, reducing available data. The third arrangement did not provide many arriving rays, only those cycling over great ocean depths, and did not provide the ray arrival patterns required for "ray differential" tomography. Thus only instruments at 1000-m depth were rigorously tested.

\section{SIMULATION METHOD}

\section{A. Propagation}

Two runs of the propagation model are required to complete a simulation. A set of reference ray-arrival times and a set of simulated observational ray-arrival times must both be generated. Reference times are denoted $T_{i j}$ and arrival times are denoted $A_{i j}$. The subscript $j$ indicates the possibility of multiple arrivals at each receiver; $i$ distinguishes receivers.

The reference times $T_{i j}$ are obtained by ray tracing through the mean layered sound-speed field, called the reference ocean. These times are associated with the eigenray paths between the sources and the nominal receiver locations $\mathbf{X}_{i}$, with the random vectors $\left(\mathbf{d}_{i}\right)$ equal to zero. Reference times are computed in the same maner for experimental ocean-ray tomography (Cornuelle et al., 1985, e.g.). The set of reference times must only be calculated once for each array configuration $\mathbf{X}_{i}$.

The simulated eigenray arrival times $A_{i j}$ are obtained by ray tracing through the total simulated ocean, reference ocean plus perturbations, from the sources to the simulated receiver locations $\mathbf{X}_{i}+\mathbf{d}_{i}$. These simulated times are data which are input to the tomographic mapping (inversion) procedure. The set $A_{i j}$ must be computed for each realization.

The $\mathbf{d}_{i}$ are generated from isotropic joint-normal distributions, with means of zero and standard deviations $\sigma_{d}$ in normal directions ( $x$ and $y$ ) varying from 0.01 to $5000 \mathrm{~m}$. The effect of the altered range greatly exceeds the effect of altered azimuth in long-range ocean propagation, so the errors are effectively only source-to-receiver range errors normally distributed with standard deviation $\sigma_{d}$. The $\sigma_{d}$ $=0.01-\mathrm{m}$ case is intended to represent perfect knowledge of receiver positions $\left(d_{i}=0\right)$ but is nonzero to allow logarithmic plotting of results. Each mapping realization uses a unique set of $\mathbf{d}_{i}$ vectors.

\section{B. Tomographic reconstruction}

The sound-speed field is mapped using established techniques of three-dimensional ocean acoustic tomography (Cornuelle et al., 1989; Cornuelle et al., 1985; Cornuelle, 1982; Cornuelle, 1985; Munk and Wunsch, 1979). Technical aspects of the propagation requirements, signal processing, travel-time data processing, and inversion procedure are covered in those and other papers.

First, propagation through a reference ocean field is forward modeled as previously described, forming $T_{i j}$. Observational arrival times of individual multipath signals, $A_{i j}$, simulated in our case, are then compared with $T_{i j}$. The differences $\left(A_{i j}-T_{i j}\right)$ are then used to generate a map of perturbation ocean structure (relative to the reference) using linear inverse theory. The sum of the reference and perturbation fields is the mapped field of ocean structure. The inverse procedure is blind to position errors.

The procedure requires that the ocean structure is wellenough known to enable characterization of arriving acoustic energy, that is, to directly relate acoustic arrivals with eigenray paths so that the components $A_{i j}-T_{i j}$ are meaningful. Ray arrivals from the reference and data-simulation runs are chosen for inversion if ray initial angles agree within a tolerance of 0.08 radians $(4.6 \mathrm{deg})$, eliminating up-down ray ambiguity and other confusion which could be eliminated by using a pair of hydrophones. Typically $80 \%$ of the reference rays are identified in the simulated data. Most of the missing $A_{i j}$ occur over the longer paths.

The generalized inverse (Aki and Richards, 1980) is used to generate a parametrized form of the perturbation field using $A_{i j}-T_{i j}$ (Cornuelle et al., 1989; Comuelle, 1982; Howe et al., 1987). A solution is sought that minimizes squared model parameters and minimizes arrival-time mismatch between data and predictions from the solution. The parametrization used in this study is a summation of vertical modes and horizontal basis functions. The horizontal functions are the first eight harmonics of a fundamental with wavelength $1000 \mathrm{~km}$, with a spectral shape consistent with a Gaussian covariance function having characteristic scale 60 $\mathrm{km}$. This horizontal basis set is similar to that used in a moving-ship tomography study (Cornuelle et al., 1989). The vertical mode functions are taken directly from the soundspeed field simulation, and are similar to those which would result from principal component analysis (or empirical or- 
thogonal function analysis) (Cornuelle et al., 1985; Tolstoy et al., 1991).

Moderate nonlinearity, in the form of ray-path differences through the reference and perturbed ocean structures, giving geometric timing deviation, can be handled with iterative application of forward modeling and inversion (Cornuelle et al., 1993). This seems attractive, since corrected receiver positions are one of the results of the first iteration (Cornuelle, 1985). For simplicity, this study uses single inversions, without iteration, therefore the solution assumes that eigenrays are not significantly displaced from their trajectories in the reference ocean. The two smaller arrays are in a regime of short propagation range, where iteration does not change or improve results for the simulated level of sound-speed perturbation; for consistency the larger array is treated in the same manner, although iteration does change the results slightly.

In a real-ocean experiment, the anomalies $A_{i j}-T_{i j}$ would have three sources: sound-speed deviations from the reference field (our signal), the random position components of the simulated receivers, and internal-wave effects. Our simulated propagation includes the first two, but does not include the internal-wave effects due to resolution limitations in the ocean model. In a limited number of cases we simulate internal-wave noise by adding Gaussian random noise to the $A_{i j}$ after propagation modeling. The position errors are nearly constant for all arrivals at each receiver, whereas the internal-wave induced noise is independent over arrival.

The inversion procedure will not produce a perturbation field (or updated map) which is exactly consistent with the simulated data unless a model (perturbation field) of unwarranted high complexity is fitted to the data. Such a highly complex model would include small-scale structures (e.g., internal waves) in the mapped field to absorb the inconsistencies in data due to noise. The vertical separation of the eigenrays and incomplete spatial ocean sampling make use of a complex model unreasonable, since details are unresolvable. It is more logical to allow the perturbation field to be slightly inconsistent with the data. Since the receiver positions are not precisely known, the full solution includes receiver displacements $\mathbf{d}_{i}$ as well as ocean perturbations. It is possible to include these displacements because they are limited in their ability to fit the data though cross relations between the $A_{i j}$ of each receiver, and because only a single $\mathbf{d}_{i}$ can effect each $A_{i j}$, reducing nonuniqueness problems. This contrasts with the inclusion of volumetric internal-wave effects, where many parameters could influence each $A_{i j}$ value, giving an exact but nonuniquely determined fit to the data.

In the simulations, smooth models of mesoscale structure and receiver displacements can be fit with reasonable error to the simulated data, consistent with these ideas. Because smooth fields are selected by the inversion, in a tradeoff with imperfect fit to the data, introduction of internalwave and other finescale-induced arrival-time errors into the simulations has little or no effect on the fitted model.

The $300-\mathrm{km}$ inversion simulation takes about $25 \mathrm{~min}$ using a Sun Microsystems Inc. Sparcstation 2 computer. The range-dependent raytrace takes $15 \mathrm{~min}$, the dependency matrix calculation takes $3 \mathrm{~min}$, the inversion takes $6 \mathrm{~min}$ and the mapping (from the model-field parameters) takes $1 \mathrm{~min}$. The reference raytrace step $(8 \mathrm{~min})$ does not need to be repeated for multiple realizations and is not included in the total, since the $T_{i j}$ are identical for all realizations using a particular array geometry.

\section{MAPPING RESULTS}

The objective of this work is to evaluate the usefulness of mesoscale maps made using simulated data of various quality. Such maps must be statistically evaluated for similarity to the simulated (known input) field. They can also be visually examined to determine their ability to find features in the field. Such features might become candidates for further study or survey in practical applications.

Maps of sound-speed uncertainty (error variance) in the estimated fields provide a guide for evaluating the quality of those fields, but do not fully quantify the usefulness of patterns which appear but have variances near the expected uncertainties. We therefore directly compute errors by comparing output maps to the simulated ocean. We wish to investigate the usefulness of patterns which appear but have variances comparable to the expected uncertainties. These may be rigorously classified as noise but may provide desired information, for example, locations of warm and cold features to further investigate.

The least-squares calculations used here (including the error maps) assume Gaussian statistics of the field and the error sources, and assume linearity of the inversion. Parameters are adjusted in the simulations such that these assumptions are satisfied, assuring that the mapping procedure is applicable, but cannot always be controlled in field work.

We evaluate maps of sound speed at 700-m depth made under a variety of conditions. The conditions varied are: (1) the array size; (2) the location of the $300-\mathrm{km}$ array; (3) the rms receiver displacements $\mathbf{d}_{i} ;(4)$ the sound-speed variance in the simulated ocean; and (5) internal-wave noise (either present or absent). The depth $700 \mathrm{~m}$ is chosen because it is away from any baroclinic mode nodes, so that all modes are useful in the inversion. It is also the depth chosen for the ASA-meeting tomography benchmark testing.

Maps of error variance for the output estimation can be generated from the receiver uncertainties and variance estimates of the modes describing the field. These mode variances can be estimated from characteristics of the data, such as the navigation and travel-time uncertainties, and from expected variances of the field. The error maps are rather insensitive to variations in $\sigma_{d}$ for all of the array shapes. An example of 700-m depth error for the default location of the $300-\mathrm{km}$ array is shown in Fig. 6. The error is shown to drop to about $1 \mathrm{~m} / \mathrm{s}$ rms within portions of the mapped area, reduced from the a priori sound-speed field standard deviation of $3.2 \mathrm{~m} / \mathrm{s}$ at $700 \mathrm{~m}$ depth (seen at the edges of the map where no data is available, and with no data the inversion maps the field to zero). This a priori value is consistent with the high-variance curve of Fig. 3, and variances of mode uncertainties are properly scaled for comparison with the originally provided simulated field.

Four quantities are calculated to estimate the quality of the resulting field estimates. These each highlight different 

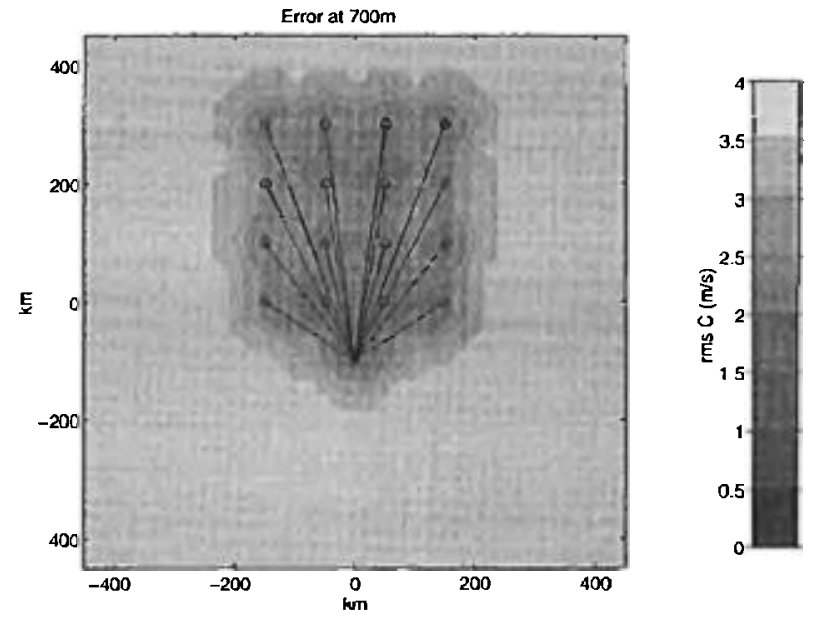

FIG. 6. The map of rms error for the $300-\mathrm{km}$ grid with receiver uncertainty of $\sigma_{d}=1-\mathrm{km}$ rms. The error is about $3.2-\mathrm{m} / \mathrm{s}$ rms outside the array, consistent with assigning no perturbation to the regions from which no data is laken, so that the error is comparable to the expected (a priori) variance of the field. It drops below $1-\mathrm{m} / \mathrm{s}$ rms near a few of the receivers (obscured in the figure). The drifter array and the source are indicated with circles. Acoustic paths are shown, diverging from the source.

aspects of the match (or mismatch) between the known and the estimated field. These quantities are computed over the area sensed by the array, essentially the area of square receiver array, but extending also to the source. They are the following.

(1) Correlation coefficients, or normalized unlagged covariances of the two fields.

(2) The variance of the mapped field, divided by the variance of the simulated field. The mapped field will have low value if the field is not effectively sampled, and can have a high value if noise has similar characteristics to signals that would appear from coherent features. This can be ambiguous in meaning, and alone does not provide sufficient information to judge maps.

(3) The mean difference between the two fields, or the bias.

(4) The variance of the simulated (input) field minus the estimated field, normalized by the variance of the simulated field. This is the percentage of variance unaccounted for by mapped structures, the residual variance, a measure common in analysis of fitting.

\section{A. Effect of array size}

The receiver array sizes, 150,300 , and $700 \mathrm{~km}$ square, each with 16 gridded receivers, show differing performance. The 300-km array does the best [Fig. 7(a) and (b)] because the horizontal interpath spacing is small enough that horizontal coherence of the mapped field, forced by the limited number of horizontal harmonics, allows for effective interpolation into the gaps between paths. The $150-\mathrm{km}$ array also shares this feature, and its reduced performance is due in part to the specific features in its domain, since it is rigorously tested at only one position. The larger 700-km array has larger unsampled spaces, of size comparable to the $100-\mathrm{km}$ eddy decorrelation scale. It has no mapping capabilities in these gaps, and does not perform as well as the other two.

The 300 - and $150-\mathrm{km}$ arrays are comparable in perfor-

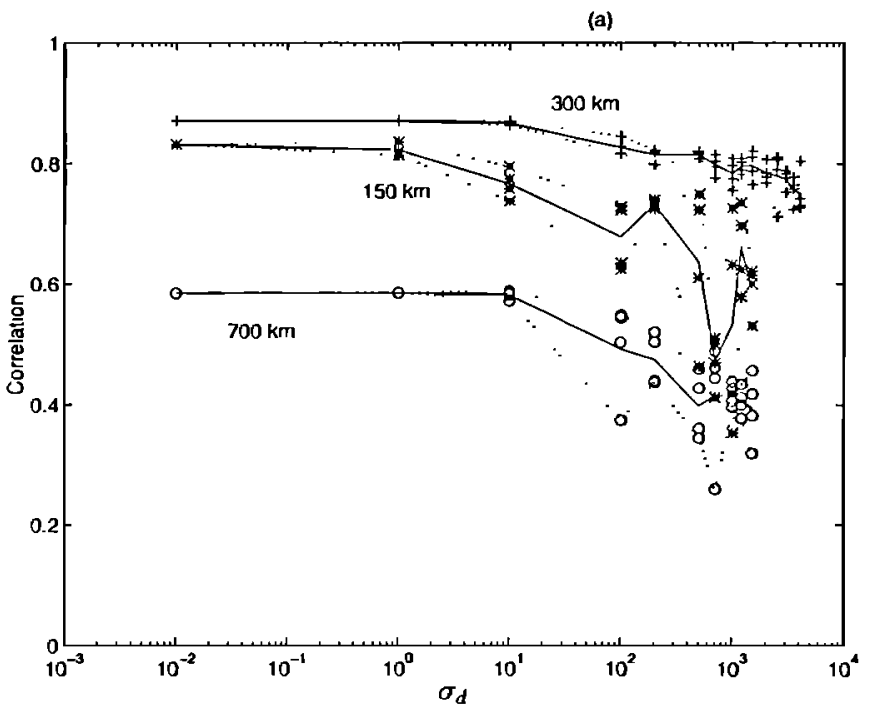

(b)

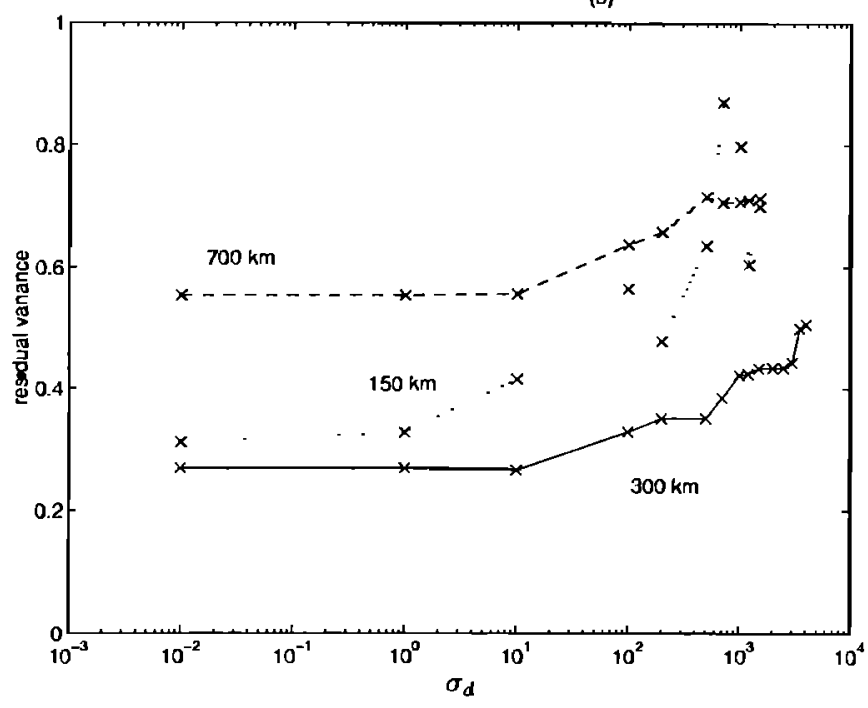

FIG. 7. (a) The correlation coefficients for many of the tomography runs are indicated by the symbols: +'s for the 300-km array. *'s for the $150-\mathrm{km}$ array, and $O$ 's for the $700-\mathrm{km}$ array. These are from cross products computed over the domains well measured by each array. The lines show the means over all realizations for each array- $\sigma_{d}$ combination, and the dotted lines indicate the maximum and minimum values for each array. The $300-\mathrm{km}$ array shows the highest correlation. The correlation begins to diminish for rms displacements greater than about $200 \mathrm{~m}$. especially for the small array, but stays quite high even for km-scale displacements for the $300-\mathrm{km}$ array. (b) The mean residual variances show the same general behavior as the correlation coefficients: The $300-\mathrm{km}$ array extracts most of the variance of the field, and the mapping ability deteriorates for $\sigma_{d}$ greater than approximately $200 \mathrm{~m}$.

mance, within the limits of reliability of this study. Since the $150-\mathrm{km}$ array covers only one-quarter of the area with the same number of instruments, with no increase in performance, it is not as effective. The spatial oversampling with the smaller array does not improve the maps.

The horizontal data-density gain of the smaller array is actually reduced somewhat (from the geometric factor of four) because more of the source-receiver pairs are in shadow zones associated with convergence zones. The usable quantity of ray paths is slightly less than for the $300-\mathrm{km}$ array. A general observation can be made. The data density 

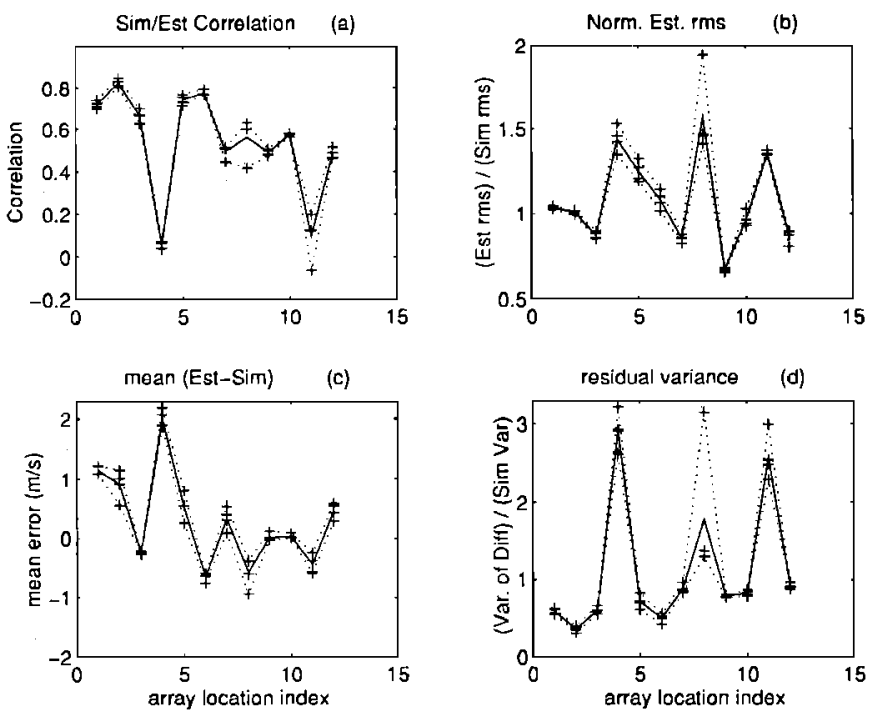

FIG. 8. Four measures of mapping performance are shown for 12 positions of the $300-\mathrm{km}$ array. The baseline position results are not shown here. The performance is variable, and depends on the characteristics of the field at each position. (a) The correlation coefficient is near 0.8 for four positions, but drops below 0.2 for two positions, 4 and 11. (b) The fitted field is far more energetic than the simulated ocean for the two cases of low correlation, plus one other. (c) The mean value of the fitted field is also variable, with largest error (bias) for the poorly fitting position 4 . (d) The residual variance, computed after the fitted field is subtracted from the simulated ocean (input field), is below the input-field variance except for poorly fit positions 4,8 , and 11 .

per unit area will generally be increased by a factor between one and $M$ (rather than the simple geometric factor $M$ ) as a square array is reduced in area by a factor of $M$, if the smaller array spans only a few convergence zones. The effects of the sound channel, convergence zones, and attenuation by the seafloor will effect the data density in different ways for differing environments.

\section{B. Sensitivity to array location}

The $300-\mathrm{km}$ array is now tested at 13 locations within the 500 - by $500-\mathrm{km}$ domain. This provides a test of its ability to map various features. Inversions are simulated with only one value of receiver uncertainty, $\sigma_{d}=1-\mathrm{km}$ rms. The baseline location of the $300-\mathrm{km}$ array, position 13 , is between -150 and $150 \mathrm{~km}$ in $x$, and between 0 and $300 \mathrm{~km}$ in $y$. In this test, locations 1 through 4 are offset $100 \mathrm{~km}$ in $y$ (to the "north"), and moved -300 (location 1), $-100,100$, and 300 $\mathrm{km}$ (location 4) in $x$. Locations 5 through 8 are offset -200 $\mathrm{km}$ (to the "south") from locations 1 through 4 , with 9 through 12 offset $-200 \mathrm{~km}$ further. The locations are shown in Fig. 5(b).

Figure 8 shows statistics of the resulting maps. Two locations (4 and 11) produce poor maps, with zero or even negative correlation with the known field. These produce difference variances in excess of the original field variances, and give no variance reduction [Fig. 8(d)].

The map from position 8 has high variance, providing large residual variance if subtracted from the starting field, but the estimate is correlated with the true field, indicating that the major features are of the correct sign. The remainder
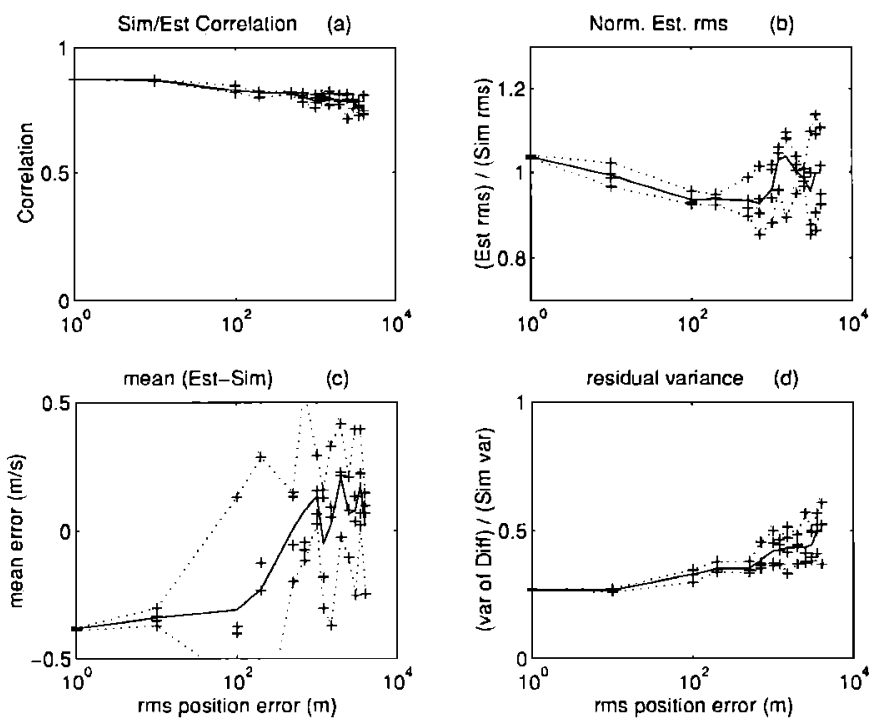

FIG. 9. Four measures of mapping performance are shown for the $300-\mathrm{km}$ array at the baseline position, as functions of $\sigma_{d}$. The dotted lines outline the maxima and minima of the realizations, shown by + 's. The solid lines show the means. The correlation (a) remains high, but the residual variance (d) increases above $\sigma_{d}$ of $500 \mathrm{~m}$. The bias (c) is the most variable property, starting out negative and moving towards zero as $\sigma_{d}$ increases. The normalized variance of the estimated field first decreases, then increases for the higher $\sigma_{d}$ cases. The data in (a) and (d) are also shown in Fig. 7.

of the runs provide both significant variance reduction and good correlation.

\section{Effect of receiver position uncertainty}

Unknown receiver positions degrade the field estimates, with errors in the maps growing with position uncertainty. Figure 9(a) shows the correlation functions decreasing as $\sigma_{d}$ increases from less than $1 \mathrm{~m}$ to over $1 \mathrm{~km}$. Understandably, residual variance increases with $\sigma_{d}$ [Fig. 9(d)]. Figure 7(a) and (b) show this also. Four realizations are performed at each $\sigma_{d}$.

Very small position uncertainties, $\sigma_{d}<1 \mathrm{~m}$, always yield the same inversion output because the acoustic data are never considered to be perfect. The assumed noise variance of the acoustic sensing system is $10(\mathrm{~ms})^{2}$, so maps are not selected to match the data perfectly, but are the smoothest which fit within the expected measurement uncertainty. Therefore precise navigation merely exposes other sources of error, such as poor horizontal coverage of the array, poor arrival-time resolution, or internal-wave noise, which are practical limitations not associated with $\sigma_{d}$.

The residual variance should saturate at large $\sigma_{d}$. At this point, the inverse is using only arrival-pattern information, since the receiver positions input to the estimation are different enough from the true positions to eliminate any effective use of the actual travel times for mean sound-speed estimation. Further increase of $\sigma_{d}$ will not reduce map quality until ray identification problems at very large $\sigma_{d}$, make mapping impossible. Before the saturation point is reached, residual variance grows with $\sigma_{d}$, since mean sound-speed information in the arrivals decreases with increasing $\sigma_{d}$. For the 300- and 700-km arrays, Fig. 7(b) shows that saturation oc- 

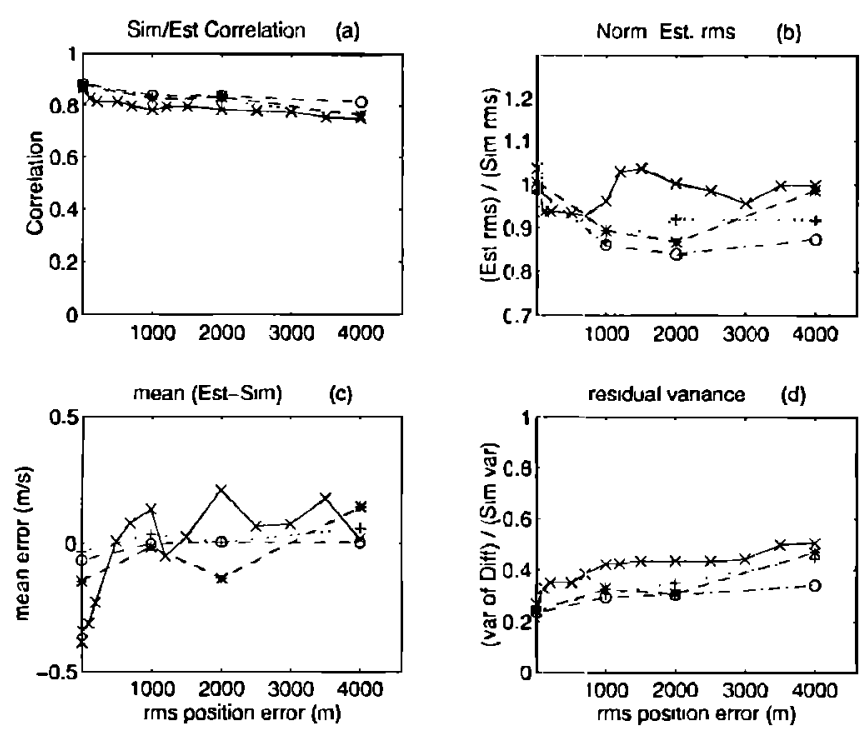

FIG. 10. The means of the residual variances for the mappings of fields with four different sound-speed variances are plotted togt:ther for comparison. All are for the baseline position of the $300-\mathrm{km}$ array. The $X$ 's and the solid line show the results for the full-variance mappirg (dat 1 of Fig. 9), the *'s and the dashed line show the $\frac{1}{4}$-variance results. the O's and the dash-dot line show the $\frac{1}{16}$-variance results, and the $t$ 's and the dolted line show the $\frac{1}{64}$-variance results. The reduced-variance fields are mapped with slightly less residual variance, but the reduction sh.sus no clear trend with field variance.

curs at $\sigma_{d}=1 \mathrm{~km}$, with the region of increasing residual extending from $10 \mathrm{~m}<\sigma_{d}<1 \mathrm{~km}$.

\section{Effect of eddy strength}

The variance of sound speed in the simulated ocean is altered (reduced) in order to evaluate the ability of the drifting receivers to map ocean features of differing intensity. There is slight improvement of the estimates, evaluated statistically, although no internal-wave noise is included. This means that nonlinearity has a slight effect at the highest variance, but not enough to significantly degrade the maps. This separation of field strength and internal-wave noise is somewhat artificial. If internal wave noise were to be included, then weaker fields would be more difficull to map than stronger ones, since they would have small signal-to-noise ratios, so this test of linearity does not demonstrate that weak eddy fields would be easier to map in the field because the inversion would be more linear, only that errors from nonlinearity would be reduced.

Figure 10 shows the mean results for mapping of the reduced variance fields, without showing the individual realization results. They can be directly compared to Fig. 9. Frame (d) shows the mean residual variarces. The slight improvement of performance (reduced resiclual) for the lower variance fields is seen, but the effect is slight compared to the complete degradation of maps in certain locations, a problem already discussed in Sec. IV A.

\section{E. Effect of internal waves}

Internal wave-induced random arrival-time perturbations have no distinct influence on map quallity. This is tested by adding small perturbations, commensurate with internalwave effects, to the travel times derived by ray-tracing through the simulated ocean. The receivers again have uncertain locations, with $1<\sigma_{d}<4000 \mathrm{~m}$. The $300-\mathrm{km}$ array is used at the baseline site. Statistical analysis of the mapped fields gives results similar to those in Fig. 9.

This insensitivity results from the inverse procedure not finding a solution exactly fitting the data. The maps should lack sensitivity to internal-wave travel-time errors within the expected variance. This variance has been both modeled (Flatté and Stoughton, 1986) and measured (DeFerrari et al., 1983; Duda et al., 1992; Stoughton et al., 1986), so that properly accounting for it in the inverse is not difficult.

\section{SUMMARY}

The mapping simulations show that receiver position uncertainties of hundreds of meters do not seriously degrade map quality for midlatitude, deep-ocean applications. Ignorance of the precise travel time does not make mapping impossible, as previously noted by both Gaillard (1985), who considered a single source/ruceiver pair, and Cornuelle (1985), who considered only the 1981 experiment geometry. The simulations help quantify' our knowledge that some acoustic remote sensing information is contained in the patterns of multipath signals, as opposed the actual times-offlight. Features in the maps change by only small quantitative amounts as position errors increase to $1000-\mathrm{m} \mathrm{rms}$, and the changes are qualitatively difficult to distinguish.

Each mapping simulation used a single source and sixteen receivers. It is informative to point out that better mapping results could have been achieved with a larger number of receivers.

For our restricted set of array geometries, the $300-\mathrm{km}$ array has the most appropriate size for successfully mapping the simulated ocean structure. This is because its interpath distances are small enough to provide mesoscale feature resolution. but are large enough so that each path provides independent data. In addition to having redundant data, the small array was a bit inferior to the $300-\mathrm{km}$ array because the number of multipaths was small, yielding little information in the absence of precise navigation. Determination of effective array geometry for a particular experiment can be done by considering the Rossby radius of deformation (Gill, 1982), topography, and eddy dynamics for the region.

Although the changes are usually small, map reliability decreases with decreased navigation accuracy, as expected. The slight reduction in accuracy can be seen in Fig. 11, which shows three maps corresponding to simulated receiver displacements of 1-, 200-, and 1500-m rms for the $300-\mathrm{km}$ array. Reliability decreases as position uncertainty increases from 10 to $1000 \mathrm{~m}$, then performance stabilizes (saturation).

We see that if the array is "optimally" positioned to sense structure, which isn't always the case for a finite set of sources and receivers, then precise navigation does not improve maps much over 500-m navigation. Figure 12 shows good-quality maps generated at three locations of the $300-\mathrm{km}$ array. Figure 13 shows three maps at poorperforming array locations. 

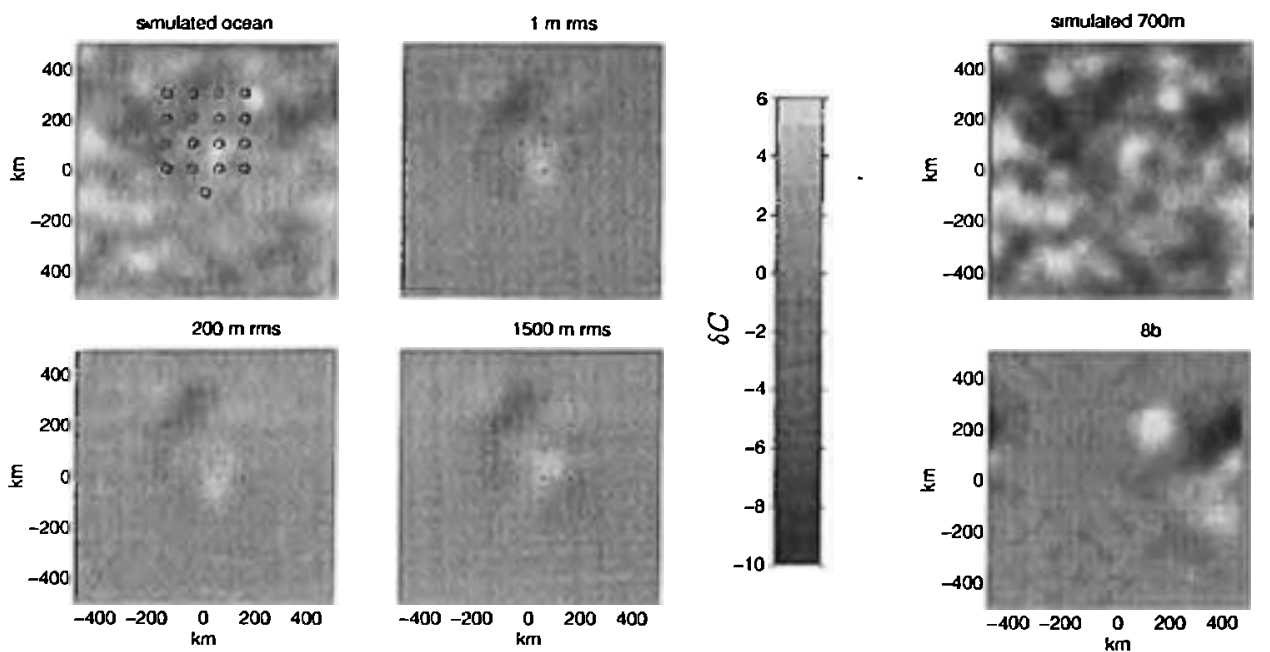

$\circlearrowleft-2$

$8 b$

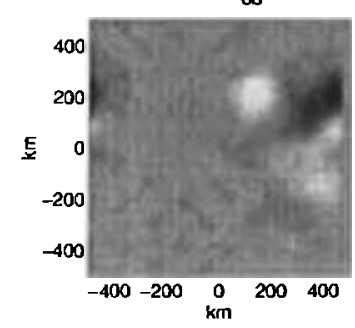

$4 b$

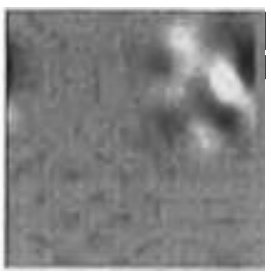

Bo

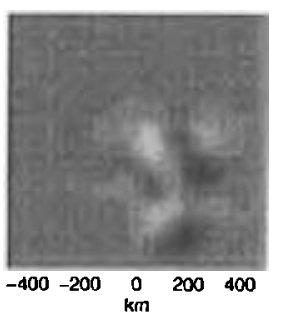

FIG. 13. Mapping realizations at $700-\mathrm{m}$ depth with $\sigma_{d}=1000 \mathrm{~m}$ are shown for positions 4,8 , and 11 of the $300-\mathrm{km}$ array. Viewing this figure and looking at the information in Fig. 8 shows that these maps are poor reproductions of the tield. for the baseline position of the $300-\mathrm{km}$ array. The maps are shown to replicate the low sound-speed feature in the upper-left portion and the highsound-speed feature in the lower-right portion of the measured domain of the simulated ocean (top-left frame). The maps change little as the receiver uncertainty $\sigma_{d}$ increases from $1 \mathrm{~m}$ to $1500 \mathrm{~m}$. The "ghosting" away from the mapped area is caused by sidelobes of the covariance function of the fitted field. caused by the finite spatial bandwidth of the spectral representation. The sidelobes are small and appear primarily when there is no other information.

None of our simulations achieves the performance of moving-ship tomography (MST) with navigated, moored sources and partially navigated, ship-lowered receivers (Cornuelle et al., 1989). The MST simulations with order onekilometer position uncertainty show comparable errors, but only one geometrical array configuration was investigated. The simulation analysis work in this paper complements the MST work in a straightforward way. We have shown that the quality of maps resulting from numerous peripheral stations, the geometry of the MST study, is attainable with a few drifting receivers, provided that the receivers are spaced two dimensionally in a manner consistent with proper sampling of the dominant sound-speed variability scales. Rapidly de-

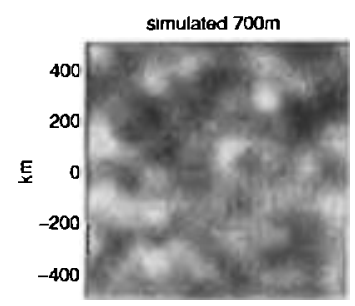

$3 b$

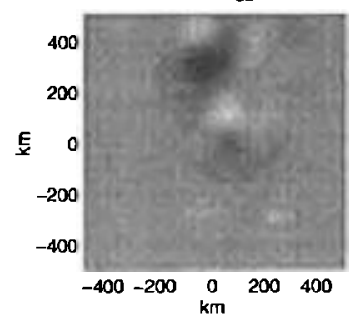

$2 b$

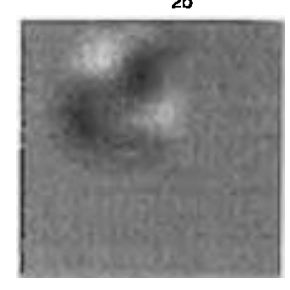

$6 b$

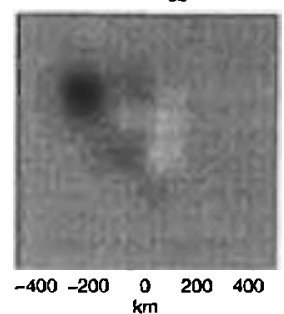

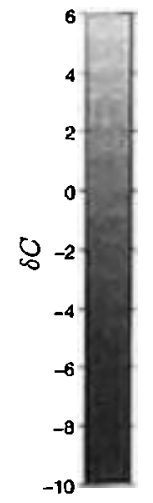

FIG. 12. Mapping realizations at 700-m depth with $\sigma_{d}=1000 \mathrm{~m}$ are shown for positions 2, 3, and 6 of the 300-km array. Viewing this figure and looking at the information in Fig. 8 shows that these maps contain the major features in the domains and account for much of the variance of the fields. ployable, radio-link receivers would have the advantage of concurrent measurement of the entire domain, unlike the MST situation where the data is sequentially collected over the domain by a small number of ships.

A plausible method for improving the tomographic scheme would be to use receiver tracks obtained from sequential transmissions. The receiver positions are deducible to within tens of meters, allowing velocity determinations to within about $1 \mathrm{~cm}$ per second over an hour. These receiver velocities, if geostrophic, should combine coherently with tomographically sensed hydrography to improve the maps.

\section{ACKNOWLEDGMENTS}

This work was supported by Office of Naval Research grants NOOO14-91-J-1138 (Arctic Sciences) and NOOO14-92J-1162 (Ocean Acoustics). The journal reviewers provided a few helpful pointers. This is WHOI contribution number 8969.

Aki, K., and Richards, P. G. (1980). Qutuntitative Seismology: Theory and Methods (Freeman, San Francisco).

Chester, D., Malanotte-Rizzoli, P., Lynch, J. and Wunsch, C. (1994). "The eddy radiution field of the Gulf Stream as measured by ocean acoustic tomography." Geophys. Res. Letl. 21, 181- 184.

Chiu, C-S., and Desaubies, Y. (1987). "A planetary wave analysis using the acoustic and conventional urrays in the 1981 ocean acoustic tomography experiment." J. Phys. Oceanogr. 17, 12791287.

Cornuelle, B., Munk, W. and Worcesier, P. (1989). "Ocean acoustic tomography from ships," J. Geophys. Res. 94, 6232-6250.

Comuelle, B., Wunsch, C. Behringe, D. Birdsall, T. Brown. M. Heinmiller. R. Knox, R. Met/ger, K. Munk, W. Spiesberger. J. Spindel, R. Webb, D. and Worcester. P. (1985). "Tomographic maps of the ocean mesoscale. Part I: Pure acoustics," J. Phys. Oceanogr. 15, 133-152.

Comuelle, B. D. (1982). “Acouslic tomography." IIEEE Trans. Geosci. Rem. Sens. GE-20. 326-332.

Comuelle, B. D. (1985). "Simulations of acoustic tomngraphy array performance with untracked or drifting sources and receivers," J. Geophys. Res. 90. 9079-9088.

Cornuelle, B. D., Worcester, P. F. Hildebrand, J. A. Hodgkiss, W. S., Jr., Duda, T. F. Boyd, J. Howe, B. M. Mercer, J. M. and Spindel, R. C. (1993). "Ocean acoustic tomography at 1000-km range using wavefronts measured with a large vertical array," J. Geophys. Res. 98. 16.365-16,377. 
DeFerrari, H. A.. Davis, R. I. Nguyen, H. Tusti 1g, २. F. and Williams N. J. (1983). "Measurements of transmission fluctuations at three ranges for refracted paths through the deep ocean," J. Acol st. Soc. Am. 74, 14481463.

Duda, T. F., Flatté, S. M. Colosi, J. A. Cornuclle, B. D. Hildebrand. J. A. Hodgkiss, W. S.. Jr., Worcester. P. F. Howe, B M. Mercer, J. M. and Spindel, R. C. (1992). "Measured wavefront fluctuations in 1000-km pulse propagation in the Pacific Ocean," J. Ac sust. Soc. Am. 92, 939-955. Dushaw, B. D., Worcester, P. F. Comuelle, E. D. a ld Howe, B. M. (1994). "High-frequency ( $>1 \mathrm{cpd}$ ) travel time variability of long-range recipmal acoustic transmissions in the western North A lanic," J. Acoust. Soc. Am. 96. 3236(A).

Flatté, S. M., and Stoughton, R. B. (1986). "Theory of acoustic measurement of internal wave strength as a function of depth, horizontal position, and time", J. Geophys. Res. 91, 7709-7720.

Gaillard, F. (1985). "Ocean acoustic tomography with moving sources or receivers," J. Geophys. Res. 90, 11,891-11,898.

Gill, A. E. (1982). Atmosphere-Ocean Dynumics (A.cademic. Orlando).

Howe, B. M., Worcester, P. F. and Spindel. R. C. (1987). "Ocean acoustic tomography: Mesoscale velocity," J. Geophys. Rıs. 92, 37853805.
Lynch, J., Pawlowicz, R. Duda, T. and Newhall, A. (1992). "Relative arrival tomographic reconstructıon using drifting receivers and a ship-board source," J. Acoust. Soc. Am. 92, 2324(A).

Munk, W. H., and Wunsch. C. (1979). "Ocean acoustic tomography: A scheme for large-scale monitoring," Deep-Sea Res. 26A, 123-16I.

Rossby. H. T., Dorson. D. and Fontaine, J. (1986). "The RAFOS System," J. Atmos. Ocean. Technol. 3, 672679.

Send, U., Schott, F. Gaillard. F. and Desaubies, Y. (1995). "Observation of a deep convection regime with acoustic tomography," J. Geophys. Res. 100. 6927-6941.

Stoughton, R. B.. Flatté, S. M. and Howe, B. (1986). “Acoustic measurements of internal-wave rms displacement and rms horizontal current off Bermuda in late 1983," J. Geophys. Res. 91. 7721-7732.

Tolstoy, A., Diachok. O. and Frazer. L. N. (1991). "Acoustic tomography via matched field processing," J. Acoust. Soc. Am. 89, $1119-1127$.

Worcester, P. F., Lynch, J. F. Morawitz. W. M. L. Pawlowicz, R. Sutton. P. J. Cornuelle, B. D. Johannessen. O. M. Munk, W. H. Owens, W. B. Shuchman. R. and Spindel, R. C. (1993). "Evolution of the large-scale temperature field in the Greenland Sea during 1988-1989 from tomographic measurements," Geophys. Res. Lett. 20, 2211-2214. 